Discussion Papers of the

Max Planck Institute for

Research on Collective Goods

2020/21

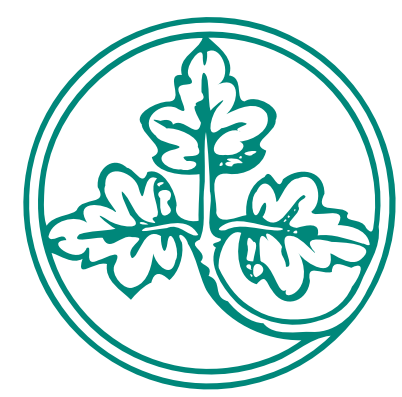

Psychological pressure and the right to determine the moves in dynamic tournaments - Evidence from a natural field experiment

Mark Kassis

Sascha L. Schmidt Dominik Schreyer

Matthias Sutter 


\section{Psychological pressure and the right to determine the moves in dynamic tournaments - Evidence from a natural field experiment}

Mark Kassis / Sascha L. Schmidt / Dominik Schreyer / Matthias Sutter

August 2020 


\title{
Psychological pressure and the right to determine the moves in dynamic tournaments - Evidence from a natural field experiment ${ }^{\sharp}$
}

\author{
MARK KASSIS, SASCHA L. SCHMIDT, DOMINIK SCHREYER \\ AND MATTHIAS SUTTER ${ }^{*}$
}

\begin{abstract}
In this paper, we show that the right to determine the sequence of moves in a dynamic team tournament improves the chances of winning the contest. Because studying dynamic team tournaments - like R\&D races - with interim feedback is difficult with company data, we examine decisions of highly paid professionals in soccer penalty shootouts and show that teams whose captains can decide about the shooting sequence are more likely to win the shootout. So, managerial decisions matter for outcomes of dynamic tournaments and we discuss potential reasons for this finding.
\end{abstract}

JEL codes: C93, D00, D81, D91, Z20

Keywords: Dynamic tournament, sports professionals, psychological pressure, value of decision rights, penalty shoot-outs, behavioral economics.

This version: 23 August 2020

\footnotetext{
\# We would like to thank two referees and an associate editor for excellent comments. Financial support from the Deutsche Forschungsgemeinschaft (DFG, German Research Foundation) under Germany's Excellence Strategy - EXC 2126/1-390838866 is gratefully acknowledged.

${ }^{*}$ Kassis, Schmidt and Schreyer: Center for Sports and Management (CSM), WHU Otto Beisheim School of Management, Erkrather Str. 224a, 40233, Düsseldorf, Germany (mark.kassis@whu.edu; sascha.schmidt@whu.edu; dominik.schreyer@whu.edu); Sutter: Max Planck Institute for Research on Collective Goods, Kurt-Schumacher-Straße 10, 53113 Bonn, Germany (matthias.sutter@coll.mpg.de), and University of Cologne and University of Innsbruck.
} 


\section{Introduction}

Tournaments between teams are a standard tool in managerial practice to elicit high effort levels (Konrad, 2009). Such team tournaments may take place within companies, but also across companies. ${ }^{1}$ They are often dynamic in nature in the sense that competing teams get interim feedback on how they perform in comparison to their competitors. As a consequence, competitors can immediately figure out whether they are lagging behind or are in the lead.

When several teams compete, some of them may be forced to drop out in the course of the tournament when their prospects to succeed look bad. This means that in such situations it is important to perform well also in intermediate evaluations in order to increase the chances to be able to move forward and stay in the race. As a consequence, managerial skills of team managers are very important as managers have to decide about the sequence of actions within their team in such dynamic tournaments. This is all the more important as interim feedback about the relative performance compared to competitors makes an efficient allocation of tasks to team members crucial in determining the tournament's interim standing and thus the chance of staying in the race and ultimately winning the tournament. Therefore, it seems important to examine whether managers are capable of making sophisticated decisions when given the chance to decide on the sequence of moves in a dynamic tournament.

While our economics profession would be most interested in the performance-impairing effects of psychological pressure from interim scores in tournaments that take place in a traditional economic context it is very difficult to get reliable and unambiguous data from such economic contexts. This is primarily because the necessary data are the property of the respective companies, but also because performance in a business context is often multidimensional and therefore hard to quantify unambiguously.

For this reason, lots of the literature on dynamic tournaments has used publicly available data from sports, in particular based on the assumption that professional athletes or sports coaches and managers are not inherently different from managers of firms. Apesteguia and

\footnotetext{
${ }^{1}$ Historical examples for such research tournaments would be the contest to select an engine for the firstever passenger line between two British cities, which was sponsored by Liverpool and Manchester Railway in 1829 (Fullerton and McAfee, 1999) or the "Golden Carrot Contest" in the early 1990ies when electric utilities offered a 30 million dollar reward for the first company to build an environmentally friendly refrigerator without chlorofluorocarbons (New York Times, 1992).
} 
Palacios-Huerta (2010) have analysed penalty shootouts in European football, i.e., soccer. Such shootouts are necessary when there is no winner after the regular match time, plus overtime, but when a winner must be determined to proceed with the whole tournament. In a shootout, one team starts with a kicker who tries to score a goal from 11 meters towards the goal where the competitor's goalkeeper tries to save the kick. The two teams take turns in kicking (and in whose goalkeeper is in the goal). The outcome of each kick is unambiguous, because it is either a goal or no goal. This implies that after each kick the interim score is immediately known to everybody and it is clear which team is in the lead or lagging behind. The order of kicks may therefore matter, as it has an influence on the interim scores. Lagging behind may put so much pressure on kickers that they start chocking (Dohmen, 2008; Ariely et al., 2009), for which reason the order of kicks - and thus more generally the sequence of moves in a dynamic tournament - may become important.

Apesteguia and Palacios-Huerta (2010) have reported that kicking first increases a team's chances of winning significantly. Given that about $70 \%-75 \%$ of kicks are converted as goals, kicking first makes it more likely to be in the lead - and thus more likely for the competing team to lag behind in the interim score. The competing team may therefore start chocking under the pressure to catch up, which - according to Apesteguia and Palacios-Huerta (2010) - explains their lower likelihood of winning the tournament which creates a first-mover (i.e., first-kicker) advantage in such a dynamic tournament. While such an interpretation sounds intuitive and plausible, subsequent work in soccer - as well as in other types of sports - has not been able to support the existence of a first-mover advantage. Kocher et al. (2012) have found no significant differences between first-kicking and second-kicking teams in soccer penalty shootouts in a sample that is four times as large as the one of Apesteguia and PalaciosHuerta (2010), even though first-kicking teams win slightly more often than in 50\% of cases (see also Arrondel et al., 2019). In ice hockey, taking the first shot in a shootout does not yield a significant first-mover advantage either (Kolev et al., 2015), nor does it in basketball (Feri et al., 2013). In cricket, batting first may also be a disadvantage, again speaking against a simple first-mover advantage (Bhaskar, 2009).

There are two noteworthy features of this debate about potential first-mover advantages in team sports, however. First, in interactive situations with a kicker and a goalkeeper it is 
not unambiguous what it means to move first. The existing literature assumes that taking the first kick in a soccer shootout (or the first shot in an ice hockey shootout) defines the first mover. Yet, one could also argue that a team with an exceptional goalkeeper takes the first move by letting the other team kick first so that the exceptional goalkeeper can save the kick and put the competing team in a disadvantageous position as early as possible. ${ }^{2}$ Second, since July 2003, it is endogenous which team takes the first kick in a soccer penalty shootout, meaning that one of the teams (that wins the toss of a coin) has the right to choose whether to kick first or second. This latter aspect allows us to address the question whether team managers (or teams' captains) can benefit significantly from the right to determine the sequence of moves in a dynamic tournament with interim feedback. Our approach puts the existing literature on first-mover advantages in teams sports into a new perspective, as it takes into account a stage of the tournament that precedes even the first kick, namely the stage in which one team can decide about the sequence of moves. ${ }^{3}$

We address our research question of whether the right to choose the sequence of moves in a dynamic tournament has managerial value by compiling a unique and novel dataset that is based on accessing video footage of penalty shootouts in the most important international soccer competitions for club and national teams. Watching videos is the only way of examining our question because the rules of football (as laid out in FIFA.com, 2017) do not

\footnotetext{
${ }^{2}$ Only in sports where the task is an individual, non-interactive, decision making task - like when basketball players shoot free-throws (Feri et al., 2013) - it is completely clear that the team that takes the first shot is the first mover, because the other team has no means to interfere with this shot. In sports where kickers shoot at a goal with a goalkeeper, this is not the case.

${ }^{3}$ Bhaskar (2009) studies the choice of cricket teams whether to bat first or to field first, and finds that these choices are often of poor quality, actually reducing the likelihood to win. In our view, there are fundamental differences between the choice given in cricket to that prior to a shootout in soccer. In cricket, the decision is dependent on factors of the game that go beyond the skills and resources of the captain's team, mainly, 1) weather and 2) the condition of the pitch. The weather conditions across the day(s) (e.g., wind, rain forecasts) are taken into consideration as they have a huge influence on proceedings. For the pitch, if it is soft, this favors spin bowlers, if a pitch is hard, this favors hard bowlers, and the pitch conditions deteriorate across the length of the match. So depending on these factors, and taking them into consideration together with the own team's strengths vs. the opponent's strengths, it may be advantageous for a captain to start or not. Furthermore, the deterioration of the pitch is often a huge factor influencing teams to prefer to bat first. Once the pitch is deteriorated, the ball can fly all over the place and therefore becomes harder to bat. The soccer penalty shootout is, as we argue, a lab like setting that reduces the chances of winning to nothing else but the skills and resources of players. There are no extraordinary influences on the chances of winning, and no changes in pitch conditions to consider. Therefore, only skills and resources remain, and the potential advantage of choosing the sequence of moves.
} 
require the referee to take notes of who won the toss of a coin before the penalty shootout. Only from videos one can assess whether the captain who won the toss of a coin before the shootout decided for his team to kick first (against the competing team's goalkeeper) or to kick second (meaning that the own goalkeeper starts against a kicker from the competing team). In total, we have collected data about the captains' decisions in 96 penalty shootouts, the largest database to date about how decisions of captains influence the outcome of the dynamic tournament.

We find that the team whose captain won the coin toss won in $60.4 \%$ of shootouts, which is significantly better than a $50 \%$-chance that one might expect if the captains' decisions would not make any difference and had no value for the team. There is no advantage of kicking first per se, however, since the relative winning frequency of first kicking teams is only $50.97 \%$ in international competitions (which is not significantly different from a 50:50 chance). Hence, the order of kicking does not matter, but the right to determine the sequence of moves does matter. Comparing the most important soccer competitions to less senior ones suggests that experience might contribute to the positive value of the right to determine the sequence of moves in such tournaments with interim feedback. Interestingly, we also note that only about $60 \%$ of captains decide to kick first, but about $40 \%$ prefer to kick second, thus letting their goalkeeper start. We find that the latter is more likely to be the case when their goalkeeper is taller than the opponent's goalkeeper, and less likely the case if the own team scores relatively many goals.

The rest of the paper is organized as follows. Section 2 reviews the rules of penalty shootouts and introduces our main hypothesis. Section 3 describes our data and methods. Section 4 presents our results, and section 5 concludes.

\section{Rules of penalty shootouts and hypothesis}

Penalty shootouts were introduced by the world governing body of soccer, the Fédération Internationale de Football Association (FIFA) in 1970 to determine the winner in knock-out tournament games when a match was tied after 120 minutes, including regular time (90 
minutes) plus overtime (30 minutes). Before 1970, the winner of a tied match was determined by staging another match a few days later.

The rules for a penalty shootout are as follows: First, each team nominates five players (out of the players on the pitch in the $120^{\text {th }}$ minute). The goalkeeper of each team who served at the end of the 120 minutes is automatically qualified to act during the shootout. Second, teams kick in alternating order. ${ }^{4}$ The task is to score a goal from the penalty spot that is 11 meters away from the goal. Note that the outcome of each kick is immediately resolved and transparent - either a goal converted (good for the kicker's team) or not (good for the goalkeeper's team) - which means that both teams observe the interim score instantaneously. Third, the shootout is terminated as soon as the number of penalties converted by one team cannot be matched by the other team even if the other team would convert all their remaining penalties. In case of a tie after both teams have taken five kicks, teams continue kicking in the same alternating order until one team has scored one goal more than the other team from the same number of kicks (i.e., in a sequential one-on-one competition). ${ }^{5}$

From 1970 until June 2003, the order of penalty kicks was determined as follows: before the shootout, both teams' captains could bet on a referee's toss of a coin, and the winning captain's team had to take the first kick (meaning that the losing team's goalkeeper was the first to act on their behalf). In July 2003, the FIFA changed the rules by giving the winner of the referee's toss of a coin the right of choosing whether to kick first or second. This rule change allows us to address our research question of whether having the right to determine the sequence of actions in a tournament with interim feedback has a significant value.

Recall that this right is executed by a team's captain who is often the most prominent and most experienced player in a team. This means we analyze data from highly paid professionals who act in their natural environment in a high-stakes situation. Of course, each

\footnotetext{
${ }^{4}$ In 2017, FIFA trialed a new format of penalty kicks in the 2017 UEFA European Women's Under-17 Championship finals in the Czech Republic. This format is called A-B-B-A, according to which the order of teams changes after each pair of kicks (meaning that team A starts in the first pair, but goes second in the second pair, first again in the third pair, and so forth). This rule change has, so far, only been implemented in the above mentioned junior tournament in 2017. All shootouts in our data are of the traditional format of alternating kicks (A-B-A-B-...) as described in the main text.

${ }^{5}$ A football penalty shootout belongs therefore to a class of games that Walker et al. (2011) call binary Markov games. For a theoretical account of such games, see their paper. Penalty shootouts are also a good source for empirically studying mixed strategies in games (see Chiappori et al., 2002; Bar-Eli and Azar, 2009).
} 
team has a coach who is typically responsible for selecting the first five kickers for the penalty shootout. The decision whether a team kicks first or second must be made and communicated by the team's captain, however, if he wins the coin toss before the shootout. It is impossible to determine whether the coach gives orders to his captain which choice he should make in case of winning the coin toss, and whether captains would follow these orders. Even if the latter were the case, we could still answer our main research question, namely whether having the right to determine the sequence of moves in a dynamic tournament with interim feedback has significantly positive value. Therefore, we continue by denoting the captain as making the decision about the sequence of moves. ${ }^{6}$

It seems reasonable to assume that the captain will make a choice that maximizes his team's chances to win the shootout, either by one of his team members kicking first or by having the own goalkeeper act first. We focus on the relative strength of a captain's goalkeeper to formulate our hypothesis, assuming that the (typically) five kickers of each team are, on average, equally strong, while there can be differences in a goalkeeper's skills.

If the captain's goalkeeper is considered as equally strong or worse (in saving kicks) as the competing team's goalkeeper, then we would expect the captain to decide to kick first. Since empirically the conversion rates of kickers are around $75 \%$, but goalkeepers save on average only around $15 \%$ of kicks (with the rest being kickers who miss the goal or only hit the crossbar or post; Chiappori et al., 2002), kicking first is likely to put the captain's team in the lead, and even mild psychological pressure on the competing team (because of lagging behind in the interim score) may make it optimal to kick first.

If the captain's goalkeeper is considered to be better than the competing team's goalkeeper, it may improve the chances to win the shootout by choosing to kick second. If the goalkeeper is successful in saving the competing team's first or early kicks, this may generate the first instance of a miss to score a goal for the competing team, and this first miss may have much stronger effects than later misses. Strictly speaking, what might be actually relevant is not the interim score per se (i.e., whether one is lagging behind in the current score of goals or

\footnotetext{
${ }^{6}$ See footnote 13 below for some empirical evidence that captains might not always follow their coaches' orders.
} 
not), but whether a team has missed to score a goal or not (even when missing does not mean that the score is negative). In other words, being "behind schedule" may be the decisive aspect that generates psychological pressure, rather than lagging behind in the interim score. The first instance of a miss may not only boost the own kicker's confidence to score, but may also undermine the competing kickers' confidence and let them choke more often. Then the sooner such a miss happens, the stronger the psychological pressure. This implies that captains whose goalkeepers are better than the opponent would have a chance to put very early pressure on the opponent by sending the goalkeeper first on the field (and then kick second).

From this deliberation we expect that if a captain decides to kick second, his goalkeeper should be better in saving kicks and the own kickers should be more successful in converting goals. If this reasoning applied to our data, then the likelihood to score should differ more strongly early on in the shootout between teams whose captains won the coin toss and teams whose captains lost. Overall then, the team whose captain wins the coin toss is expected to win significantly more often than in $50 \%$ of cases (with the latter being the likelihood of winning in case it was random), and this effect does not depend on the order of kicks taken. The order of kicks should only matter in combination with the captain's decision. If he decides to kick first, we should see a first-mover (i.e., first-kicker) advantage. If he decides to kick second, we should see a second-mover (i.e., second-kicker) advantage. In the following, we describe the data that we use to examine our hypothesis.

\section{Data and methods}

We have selected only international tournaments for clubs and national teams for inclusion in our data set. Since we were able to collect data on practically all important international tournaments, we consider this the most coherent criterion for data selection. ${ }^{7}$ Table 1 lists the

\footnotetext{
${ }^{7}$ With respect to national tournaments, it is much more difficult to find such a coherent criterion, because it is always somewhat arbitrary to decide which countries to include or not.
} 
14 international tournaments considered here. ${ }^{8}$ The first nine tournaments ([1] to [9] in Table 1) have the largest visibility in public, including the FIFA World Cup - both for men and for women - as most important tournament for national teams, and the UEFA Champions League as most prestigious international tournament for (European) club teams. Among the first nine tournaments, there are no junior competitions. The latter are represented in tournaments [10] to [14] and these junior competitions will serve as an important control later on. In total, our dataset provides two unique characteristics currently unaccounted for in previous penalty shootout analyses - data from both males and females and data from junior players whose career prospects depend to a large degree on the performance in these FIFA-under age tournaments (like, e.g., the U-20 FIFA World Cup).

\section{[ Insert Table 1 Here]}

From July 2003 to August 2017, there were a total of 207 penalty shootouts in all 14 tournaments considered here (see column [A] in the penultimate row of Table 1). For 206 of these shootouts, we know the order of kicks, i.e., which team took the first kick (column [B]). The large majority of these shootout data were provided by Gracenote Sports, Netherlands (Nielson Company) and other sources were used to provide supplementary data. ${ }^{9}$

The main challenge for our research question is, however, to know which team won the coin toss before the shootout and which decision was taken by the captain of the winning team. There are no public records of captains' choices in the coin toss due to there being no regulations requiring referees to record such information. Hence, to obtain these data, we requested and received (temporary) access to FIFA's and UEFA's (Union of European Football Associations) film archives and spent several days sifting through video footage to

\footnotetext{
${ }^{8}$ There are three other international tournaments that are fairly known - the Gold Cup (for national teams of North and Central America), the Asian Cup (for Asian national teams) and the Copa Libertadores (a South American international club competition). Unfortunately, we could not get access to video footage from any of these tournaments that would have allowed us to observe the outcome of the coin toss before the shootout starts. Hence, we had to exclude these three tournaments (that had comparatively few shootouts anyway).

${ }^{9}$ Email response from soccer associations, email response from soccer clubs, FIFA Films, newspaper clips, UEFA Video Archives, http://fifa.com, http://www.fussballdaten.de, http://www.kicker.de, http://scorespro.com, http://www.weltfussball.de, http://en.wikipedia.org, http://www.worldfootball.net.
} 
identify segments where it is possible to view the pre-shootout coin toss and see the referee talking to the winning captain. By knowing which captain won the coin toss and seeing which team kicked first, we can unambiguously infer the captain's decision to start either with the first kick or with the goalkeeper (and then taking the second kick).

To the best of our knowledge, we have compiled the largest sample of shootouts $(N=96)$ for which the winning captain's decision is known (see column [C] in Table 1). ${ }^{10}$ Our 96 shootouts represent $46.4 \%$ of all 207 shootouts that took place in the 14 international tournaments from July 2003 to August 2017. From Table 1, we can infer that there is no difference in the likelihood of the first kicking team to win the shootout between those shootouts for which we can identify the captain's decision and those shootouts where we do not have this information: the winning frequency of first kicking teams does not differ between the subset of 96 shootouts with video information about the captain's decision (where exactly $50 \%$ of first-kicking teams win the shootout; see column [C-1]) and the full set of 206 shootouts where we know which team kicked first (50.97\% of first-kicking teams win; see column [B-1]). In Table A1 in the Online Appendix we disentangle the shootouts with or without video footage by the stage of the tournament. We see that for the important stages - including all stages from the final to the round of last 32 - we have video footage for $64 \%$ of shootouts across these stages. While the fraction fluctuates a bit due to the small numbers of observations in single stages, in most stages we have video footage for about two thirds of shootouts, indicating that we do not have any noticeable bias in favor or against particular stages of the tournaments. Only for the very early stages of the tournaments subsumed under "Other" in Table A1 - we have only relatively little video material that allows identifying which captain won the coin toss. For the "Other" stages, we cover only $19 \%$ of shootouts with video footage. When asking for access to the FIFA's and UEFA's film archives, the UEFA legacy video team had pointed out that early stages of the

\footnotetext{
${ }^{10}$ Apesteguia and Palacios-Huerta (2010) is the only other study we are aware of that refers to the captains' decisions after the coin toss, but before the shootout. Yet, they refer only to "about twenty videos" (Apesteguia and Palacios-Huerta, 2010, p. 2554) and do not include these data in their primary analysis. Moreover, they claim that in almost all cases, the winning captains would have decided to kick first in the penalty shootout. Our much larger data set shows that their claim is not generally valid (see our results about the captains' decisions to kick first or second below).
} 
tournaments would most likely not be covered by video footage. ${ }^{11}$ This limitation has to be kept in mind in the following analysis of the data (even though our data set is the best available).

\section{Results}

Table 2 presents our main results. In panel A we show the results for tournaments [1] to [9], the nine most important international tournaments. For these nine tournaments, we know the captains' choices in 65 shootouts. Interestingly, the fraction of captains who let their teams take the first kick ( 36 out of 65 , i.e., $55 \%$ ) and of captains who want to start with their goalkeeper and then take the second kick ( 29 out of 65 , i.e., $45 \%$ ) is fairly balanced and not significantly different from a 50:50 split. These - high-stakes - decisions do not support the myth that kicking first is most important (Andersson et al., 2008), because then most captains would have chosen to let their team take the first kick. Apparently, this is not the case. The crucial question is then, are captains making good decisions.

\section{[ Insert Table 2 Here]}

From panel A of Table 2 we can see that those teams whose captains decide to take the first kick win in two thirds of cases (24 out of 36). This would be consistent with an advantage of kicking first. Yet, when we look at the outcomes for the teams whose captains decide to kick second and let their goalkeeper act first, this impression is strongly refuted. Rather, $66 \%$ of teams (19 out of 29) who take the second kick win the shootout when the captain has decided to kick second. Taken together, we note that when captains can decide whether to kick first or second, their team wins in 43 out of 65 cases $(66.2 \%)$. This winning frequency is significantly different from a 50:50 chance ( $p=0.006$; one-sided binomial test). ${ }^{12}$ This

11 In private correspondence with the first author of this paper, the UEFA legacy video team wrote: "Limitation: It is likely that qualification games are not available."

${ }^{12}$ Given our directional hypothesis, we use a one-sided test here. Even a two-sided test would yield $p=0.012$. 
main result supports our initial hypothesis and indicates that the right to choose the sequence of moves has a positive value and that captains are able to make good use of this right.

Note that if one looked only into whether taking the first kick yields a systematic advantage, then one would have observed that 34 first-kicking teams won their shootout, while 31 first-kicking teams lost it; a difference that is far from being significant. Focusing on a potential first-kicker advantage would, therefore, have disguised the value of the right to choose the sequence of kicks.

In panel B of Table 2 we consider all 14 tournaments and analyze the 96 shootouts for which we know the captains' decisions before the shootout. Here we see that 54 out of 96 captains who win the coin toss decide to kick first, while 42 want their team to take the second kick only. The relative frequencies (56\% vs. $44 \%$ ) are again not significantly different from random ( $p>0.2$, two-sided binomial test). ${ }^{13}$ However, we note once more that the right to choose the sequence of actions makes a difference. When captains decide to let their team kick first, then their team wins in 32 out of 54 cases $(59.3 \%)$, and when they let their goalkeeper start, they win in 26 out of 42 cases (61.9\%). Taken together, $60.4 \%$ of shootouts are won by the teams whose captains can decide whether to kick first or second. This fraction is again significantly different from a 50:50 chance ( $p=0.026$, one-sided binomial test). Obviously, the right to choose is important in a dynamic tournament with interim feedback. ${ }^{14}$

13 We had an opportunity to do a survey among 340 professional and amateur coaches from Germany, Switzerland and Austria at the 2017 Federation of German Football-Coaches Congress, asking them about which fraction of captains they would expect to choose to kick first. $66 \%$ of them indicated that they expected captains who won the coin toss to let their own team kick first. This fraction is higher than the actual frequency of $56 \%$ that we observe in our data (of international tournaments), but the difference is not significant. When asked what the coaches would recommend their own captain, $88 \%$ responded that they would recommend him to kick first. While these 340 coaches are a different set of coaches than the ones in our dataset, we take the difference between their recommendations and the actual behavior of captains in our dataset as suggestive evidence that captains may sometimes have a different will than their coaches, making the captains' decisions on the pitch all the more important.

${ }^{14}$ In this paper, we have taken into account only international tournaments of clubs or national teams. There are, of course, also national cup competitions where penalty shootouts determine the winner of a match. We consider these national cup competitions as less important than the international tournaments (and it is also more difficult to follow a consistent selection criterion for inclusion), and hence we have not taken into account data for national tournaments. As a kind of minor robustness check with respect to national competitions, we have looked into publicly available video footage of shootouts in the German cup competition (DFB-Pokal). In the 10 shootouts for which we could verify which captain won the coin toss, 7 times this captain's team won the shootout. While the number of observations is too low to make any meaningful claims, we simply note that 
However, the order of kicks per se is once more unimportant. Out of the 96 shootouts for which we know the outcome of the coin toss, 48 first-kicking teams win and 48 first-kicking teams lose. ${ }^{15}$

In Table 3 we corroborate our results by showing probit regressions where the dependent variable is whether a team wins the shootout $(=1)$ or not $(=0)$. In order to take into account the dependency of outcomes within a particular shootout (if one team wins, the other team must lose), we only consider one of the two teams within each shootout, more precisely the team that is mentioned first in the official match report (which corresponds to the home team unless the match is on neutral ground, in which case it is random which team is mentioned first). ${ }^{16}$

\section{[ Insert Table 3 Here]}

In column (1) of Table 3 we include only the dummy variable whether a team's captain won the coin toss (COIN-TOSS-WINNER=1). It is significantly positive, as our nonparametric tests have already shown. Then we control in column (2) for whether a team took the first kick in the shootout (FIRST-KICK=1), and an interaction term between FIRSTKICK and COIN-TOSS-WINNER (to see whether kicking first becomes important when a captain wants his team to kick first). These two variables are never significant, but COINTOSS-WINNER itself remains significantly positive (at the 5\%-level) in all regressions reported in Table 3, independent of all the additional control variables in columns (3) and (4). These include in column (3) whether a team was playing on home ground (which applies

this fraction $(70 \%)$ is very close to the respective fraction in tournaments [1] to [9] considered here $(66.2 \%)$. Note also that in these 10 shootouts, winning captains decided to kick first in 5 cases, and second in 5 cases.

${ }^{15}$ In Table A2 in the Online Appendix we corroborate the null-result with respect to the order of kicking (first or second) in the most comprehensive dataset available so far for seasons 2003/2004 to 2016/2017. We present data from 612 penalty shootouts from 24 different tournaments, both national and international ones. The set of tournaments is a strict superset of the tournaments considered in Apesteguia and Palacios-Huerta (2010) and Kocher et al. (2012), and it extends their set of tournaments by 10 additional ones. In total, there were 645 shootouts in our set and we could retrieve the order of kicking for 612 of those. We see in column [C] that in $50.16 \%$ of cases the first-kicking team wins the shootout, and in $49.84 \%$ of cases the second-kicking team. These tiny differences in winning frequencies are far away from statistical significance $(p=0.97)$.

${ }^{16}$ In principle, we can also run the same regression with both teams and adjust for clustering over shootoutID. The results reported in the main text remain qualitatively the same. 
to the host of the international tournament; HOME), dummies for a specific tournament, dummies for the different stages of a tournament (with the final as benchmark), and the size of the audience in the stadium (ATTENDANCE). None of them is significant. In column (4) we add further controls: the fraction of kickers who are defenders, midfielders, strikers or goalkeepers (POSITION); whether the captain was involved in the shootout (CAPTAIN); whether a team's coach is older than the rival's coach (COACH OLDER); the height of the goalkeeper in meters (GOALKEEPER HEIGHT); whether the goalkeeper is older than the rival's goalkeeper (GOALKEEPER OLDER); whether the team is on average older than the rival team (TEAM OLDER); and whether the team has already participated in a shootout in the same competition but an earlier stage (PRIOR SHOOTOUT). ${ }^{17}$ All except for the last variable are insignificant. The last variable is weakly significantly negative, which might hint at some reversion to the mean (if a team won an earlier shootout, it is weakly significantly less likely to win again). Yet, whichever variables we include as controls, COIN-TOSSWINNER stays positive and always significant. Hence, the right to determine the sequence of kicks is important. But why is this right so valuable?

To start with, we exploit the fact that we can split our 14 different tournaments into youth competitions (tournaments [10] to [14] in Table 1) and those tournaments with no age restriction (tournaments [1] to [9]). Arguably, captains in the latter set of tournaments have more experience and also get better information from the supporting staff about the competing team's abilities and habits (with respect to kicking penalties) than the captains in the youth tournaments who are 21 years or younger. Moreover, in the youth tournaments there are hardly any data available about the habits of kickers (with which likelihood they shoot to the right, to the left or to the middle) or the goalkeeper (diving right or left), because the players are too young for that. On the contrary, in the tournaments without age restriction,

\footnotetext{
${ }^{17}$ In a regression not reported here we also add a dummy variable whether a particular team is the "favorite" of both teams in the shootout. We classify the "favorite" as the team with the larger market value of its players. This variable might capture better skills of the favorite team. Since market values are not always available (in particular for the junior tournaments), we have fewer observations $(\mathrm{N}=81)$. Yet, most importantly, all results stay the same: COIN-TOSS-WINNER remains significantly positive, but none of the other variables is significant.
} 
a team's supporting staff meticulously collect such information and equip their players with it.

Following this line of reasoning, we expect to see a stronger advantage for coin-toss winning captains in the regular competitions (tournaments [1]-[9]) than in the youth competitions ([10]-[14]). Of the 65 shootouts in the regular competitions without age restriction, the teams whose captains could decide the sequence of moves (because of winning the coin toss) won in 43 cases, but lost in only 22 cases ( $p=0.006$; one-sided binomial test). The distribution is noticeably different in the youth competitions. There, of 31 shootouts, only 15 were won by the team whose captain decided about the sequence of actions, while the other 16 cases were lost. The difference in winning and losing frequencies between regular competitions (tournaments [1]-[9]) and youth competitions (tournaments [10]-[14]) is weakly significant $\left(\chi^{2}\right.$-test, $\left.p<0.1\right)$, supporting the notion that experience and the availability of historical data about kickers' and goalkeepers' habits and preferences matter for the value of having the right to choose the sequence of moves.

\section{[ Insert Figure 1 Here]}

Next, we take a closer look at the 65 shootouts in tournaments [1]-[9] where we see a strong influence of who wins the coin toss. Recall from section 2 that we expected captains to choose kicking first if their goalkeeper was worse or equally good as the competing goalkeeper. We can measure the fraction of kicks saved by the goalkeeper (this excludes cases where kickers miss the goal or hit the bar or post). In fact, when captains decide to kick first, their goalkeepers save $18.9 \%$ of kicks, while the competing goalkeeper saves $19.7 \%$ of kicks. These fractions are not significantly different from each other. Yet, the kickers of the team that lost the coin toss are much more likely to miss the goal or hit only the post or bar $(17.7 \%$ vs. $7.8 \% ; p<0.01)$. So, the pressure seems to be on the kickers of the coin-toss losing team who kick second and lag behind in most cases in the interim score.

The situation is quite different when captains decide to kick second and let their goalkeeper go first. We had expected that this would only be the case when the goalkeepers of the cointoss-winning captain are better than the competing goalkeeper. This is what we find in our 
data. Checking the relative frequency of saves through the goalkeeper, we note $16.4 \%$ saves for the own goalkeeper (whose captain won the coin toss), but only $9.8 \%$ saves for the competing goalkeeper. This difference is in line with our expectation and marginally significant ( $p=0.051$; one-sided $\chi^{2}$-test). The frequency of kickers missing the goal (or hitting the bar or post) does not differ between both teams and is $9 \%$ in both cases. So, if a captain decides to kick second and let the own goalkeeper start first, the goalkeeper makes the main difference and leads his team to win more often. We find supportive evidence for this statement by an additional probit regression of the captain's decision to kick second (= 1) on (i) whether the own goalkeeper is taller than the opponent's keeper (=1), (ii) whether the market value of the own goalkeeper is higher than the opponent's keeper $(=1)$, (iii) the number of goals scored (as a measure of the offensive power of the own team), and (iv) the number of goals conceded. The first two variables are positive, and the first one is marginally significant ( $p=0.088)$, supporting the interpretation that captains consider their goalkeeper's characteristics when choosing to kick first or second. The more goals the captain's team scores during regular and overtime, the more likely they are to kick first, which looks also reasonable to us.

Before concluding, we can look at the effects of winning or losing the coin toss also from a reverse angle. If winning the coin toss would not make a difference, then the scoring probability of kickers should not depend on it. In order to examine this aspect, we look at each of the kicks of the two competing teams and calculate the difference in the probability of scoring a goal between the team whose captain won the coin toss and the other team. We control for the order of kicks by looking at each kick of a particular team separately, focusing on the first five kicks (because the number of observations gets quickly very small for shootouts that continue beyond the first five kicks). Figure 1 uses the data for the nine most important tournaments (Table 2A) and shows on the horizontal axis the sequence of kicks (from first to fifth kick), and on the vertical axis the difference in scoring probabilities between the team that won the coin toss and the other team. For our final analysis, we pool the data for teams whose captains decided to kick first and those whose captains decided to kick second. Positive values on the vertical axis indicate that kickers of teams who won the coin toss are more likely to score a goal. For each single kick we note positive entries. So, 
kickers of the team whose captain won the coin toss are constantly more likely to score a goal. The difference is 10.8 percentage points for a team's first kick (see first bar). Given an overall likelihood of $72.7 \%$ to score a goal (for the first 10 kicks in a shootout), this is a strong effect of having the power to decide the sequence of actions. The differences get smaller for kicks 2 to 4 , and resurge to 12 percentage points for the fifth, and often decisive, kick.

\section{Conclusion}

Dynamic tournaments with interim feedback pose a challenge as to what is the optimal sequence of moves. Such tournaments are ubiquitous in daily life - such as on internal labor markets when candidates run for promotion or in the case of $R \& D$ races between companies. Yet, with these naturally occurring data it is difficult to measure the influence of the sequence of moves, and of the right to determine this sequence, because data are either not publicly available or performance measures are not unambiguous. In our paper, we have therefore studied the value of the right to choose the sequence of moves by accessing sports data from the most important international tournaments of European football (soccer) clubs and national teams. In these tournaments, it is often the case that a match is tied after regular and overtime, in which case a penalty shootout must yield a decision. In such a shootout, the two rival teams compete in alternating order by one team kicking from the penalty spot and the other team's goalkeeper trying to save the kick. Since 2003, the toss of a coin determines which team has the right to decide whether to start with the goalkeeper or with a kicker. The captain of the team who wins the coin toss can make this important decision. There are very high stakes involved in these decisions - moving forward in these tournaments or even winning them is extremely prestigious and important for the career of the players -, meaning that we observe highly paid professionals in a natural environment in which we can unambiguously identify their decisions and the outcomes of these decisions.

We have been able to collect the largest data set of penalty shootouts in international competitions to date. By accessing video footage of UEFA and FIFA, we could determine for 96 penalty shootouts which captain won the coin toss and what decision he made. 
Significantly different from an a priori 50:50 chance, the teams whose captains could decide whether to start with a kicker or with the own goalkeeper won in $60.4 \%$ of cases. The influence is even stronger (increasing to $66.2 \%$ ) if we only look at the nine most important tournaments (thus excluding junior tournaments). Although we have collected the largest data set that allows to study the effects of the right to choose the sequence of moves, we would like to note that the sample is still comparatively small. It would certainly be desirable to enlarge the data set in future work to get ever more precise estimates of the true effects.

Nevertheless, our results document a positive value of the right to determine the sequence of moves in a dynamic tournament with interim feedback. In fact, we can see both a firstmover advantage and a second-mover advantage conditional on whether a captain decides to kick first or kick second. In the aggregate, however, our data show that the sequence of kicks does not matter per se, an issue that has received lots of attention in the literature (Apesteguia and Palacios-Huerta, 2010; Gonzalez-Diaz and Palacios-Huerta, 2016). There is no significant advantage for teams that kick first in our dataset (similar findings are reported in Kocher et al., 2008, 2012; Feri et al., 2013; Kolev et al., 2015; Arrondel et al., 2019), as the likelihood of winning is indistinguishable from 50:50 for both first- and second-kicking teams. We think that our novel and unique dataset has provided convincing evidence that this previous literature has put the emphasis on the wrong feature. We find that the order per se is not decisive, but that it makes a difference whether managers (like football team captains) can determine who is doing which task at which stage of a dynamic tournament. This hitherto ignored aspect with respect to sports data from highly paid professionals may also be worthwhile to consider for managerial or economics research on dynamic tournaments. 


\section{REFERENCES}

Andersson, P., Ayton, P., Schmidt, C. (eds.) (2008), Myths and facts about football: The economics and psychology of the world's greatest sport. Cambridge Scholars Publishing, Cambridge.

Apesteguia, J., Palacios-Huerta, I. (2010), Psychological pressure in competitive environments: Evidence from a randomized natural experiment. American Economic Review 100:2548-2564.

Ariely, D., Gneezy, U., Loewenstein, G., Mazar. N. (2009), Large stakes and big mistakes. Review of Economic Studies 76:451-469.

Arrondel, L., Duhautois, R., Laslier, J.-F. (2019), Decision under psychological pressure: The shooter's anxiety at the penalty kick. Journal of Economic Psychology 70: 22-35.

Bar-Eli, M., Azar, O. H. (2009), Penalty kicks in soccer: An empirical analysis of shooting strategies and goalkeepers' preferences. Soccer and Society 10: 183-191.

Bhaskar, V. (2009), Rational adversaries? Evidence from randomised trials in one day cricket. Economic Journal 119: 1-23.

Chiappori, P.-A., Levitt, S., Groseclose, T. (2002), Testing mixed-strategy equilibria when players are heterogeneous: The case of penalty kicks in soccer. American Economic Review 92:1138-1151.

Dohmen, T. J. (2008), Do professionals choke under pressure? Journal of Economic Behavior \& Organization 65:636-653.

Feri, F., Innocenti, A., Pin, P. (2013), Is there psychological pressure in competitive environments? Journal of Economic Psychology 39:249-256.

FIFA.com. (2017), Fédération Internationale de Football Association (FIFA). http://www.fifa.com/.

Fullerton, R.L., McAfee, R.P. (1999), Auctioning entry into tournaments. Journal of Political Economy 107: 573-605.

González-Díaz, J., Palacios-Huerta, I. (2016), Cognitive performance in competitive environments: Evidence from a natural experiment. Journal of Public Economics $139: 40-52$ 
Harrison, G. W., List, J. A. (2004), Field experiments. Journal of Economic Literature 42, 1009-1055.

Kocher, M., Lenz, M. V., Sutter, M. (2008), Performance under pressure - The case of penalty shootouts in football. In: Myths and facts about football: The economics and psychology of the world's greatest sport. P. Andersson, P. Ayton, and C. Schmidt (eds.), Cambridge Scholars Publishing, 61-72.

Kocher, M., Lenz, M.V., Sutter, M. (2012), Psychological pressure in competitive environments: New evidence from randomized natural experiments. Management Science 58:1585-1591.

Kolev, G.I., Pina, G., Todeschini, F. (2015), Decision making and underperformance in competitive environments: evidence from the National Hockey League. Kyklos 68:6580 .

Konrad, K. (2009), Strategy and Dynamics in Contests. Oxford University Press. Oxford.

New York Times (1992), Utilities offer 30 million dollars for a better refrigerator. 8 July 1992, pp. A1, C4.

Walker, M., Wooders, J. Amir, R. (2011), Equilibrium play in matches: Binary Markov games. Games and Economic Behavior 71:487-502. 


\section{Tables}

Table 1. Shootout data from seasons $2003 / 2004$ to $2016 / 2017$

\begin{tabular}{|c|c|c|c|c|c|c|}
\hline & {$[\mathrm{A}]$} & [B] & [B-1] & [C] & [C-1] & {$[\mathrm{C}-2]$} \\
\hline Tournament & Shootouts & $\begin{array}{c}\text { Shootouts } \\
\text { with known } \\
\text { order of } \\
\text { kicks }\end{array}$ & $\begin{array}{c}\text { First- } \\
\text { kicking } \\
\text { team wins } \\
\text { (rel. freq.) } \\
\text { Subset [B] }\end{array}$ & $\begin{array}{c}\text { Shootouts } \\
\text { with } \\
\text { known } \\
\text { decision } \\
\text { of captain }\end{array}$ & $\begin{array}{c}\text { First- } \\
\text { kicking } \\
\text { team wins } \\
\text { (rel. freq.) } \\
\text { Subset [C] }\end{array}$ & $\begin{array}{c}\text { Team of } \\
\text { deciding } \\
\text { captain wins } \\
\text { (rel. freq.) } \\
\text { Subset [C] }\end{array}$ \\
\hline [1] FIFA World Cup & 10 & 10 & 0.8000 & 9 & 0.7778 & 0.5556 \\
\hline [2] European Championship & 9 & 9 & 0.4444 & 9 & 0.4444 & 0.5556 \\
\hline [3] Copa America & 11 & 11 & 0.5455 & 2 & 1.0000 & 1.0000 \\
\hline [4] Africa Cup of Nations & 13 & 13 & 0.3077 & 2 & 0.0000 & 1.0000 \\
\hline [5] FIFA Confederations Cup & 4 & 4 & 0.2500 & 4 & 0.2500 & 0.7500 \\
\hline [6] UEFA Champions League & 30 & 30 & 0.6333 & 15 & 0.6000 & 0.6000 \\
\hline [7] UEFA Europe League & 68 & 68 & 0.4853 & 13 & 0.2308 & 0.7692 \\
\hline [8] FIFA Club World Cup & 9 & 9 & 0.5556 & 7 & 0.7143 & 0.7143 \\
\hline [9] FIFA Women's World Cup & 4 & 4 & 0.7500 & 4 & 0.7500 & 0.5000 \\
\hline [10] FIFA U-20 World Cup & 20 & 20 & 0.4000 & 16 & 0.4375 & 0.5000 \\
\hline [11] FIFA U-17 World Cup & 10 & 9 & 0.6667 & 6 & 0.5000 & 0.6667 \\
\hline [12] UEFA U21 Championship & 7 & 7 & 0.5714 & 1 & 1.0000 & 1.0000 \\
\hline [13] FIFA U-20 Women's World Cup & 6 & 6 & 0.3333 & 4 & 0.5000 & 0.5000 \\
\hline [14] FIFA U-17 Women's World Cup & 6 & 6 & 0.3333 & 4 & 0.2500 & 0.0000 \\
\hline All & 207 & 206 & 0.5097 & 96 & 0.5000 & $0.6042^{\dagger}$ \\
\hline Tournaments [1] to [9] only & 158 & 158 & 0.5253 & 65 & 0.5231 & $0.6615^{\ddagger}$ \\
\hline
\end{tabular}

${ }^{\dagger} \mathrm{p}$-value $=0.026$ (one-sided binomial test)

${ }^{*} \mathrm{p}$-value $=0.006$ (one-sided binomial test) 
Table 2. Captains' decisions and outcomes for their teams

Panel A. Tournaments [1] to [9] from Table 1 - in total 65 shootouts from 2003 to 2017

\begin{tabular}{ccc}
\hline \hline & Captain decides to & Captain decides to \\
\hline Kick first & Kick second (i.e., own \\
goalkeeper starts)
\end{tabular}

\begin{tabular}{lccc}
\hline \# of shootouts & 36 & 29 & 65 \\
Team of deciding captain wins the shootout & & & 43 \\
Absolute frequency & 24 & 19 & 0.662 \\
Relative frequency & 0.667 & 0.655 & 22 \\
Team of deciding captain loses the shootout & 12 & 10 & 0.339 \\
Absolute frequency & 0.333 & 0.345 & \\
Relative frequency & & & \\
\hline \hline
\end{tabular}

${ }^{+\mathrm{p} \text {-value }=0.006 \text { (one-sided binomial test) }}$

Panel B. Tournaments [1] to [14] from Table 1 - in total 96 shootouts from 2003 to 2017

\begin{tabular}{lccc}
\hline \hline & Captain decides to & Captain decides to & \\
& Kick first & $\begin{array}{c}\text { Kick second (i.e., own } \\
\text { goalkeeper starts) }\end{array}$ & Sum \\
\hline \# of shootouts & 54 & 42 & 96 \\
Team of deciding captain wins the shootout & 32 & 26 & 58 \\
$\quad$ Absolute frequency & 0.593 & 0.619 & $0.604^{\dagger}$ \\
Relative frequency & & & 38 \\
Team of deciding captain loses the shootout & 22 & 16 & 0.396 \\
Absolute frequency & 0.407 & 0.381 & \\
Relative frequency & &
\end{tabular}

${ }^{\dagger} \mathrm{p}$-value $=0.026$ (one-sided binomial test) 
Table 3. Determinants of winning a penalty shootout

\begin{tabular}{|c|c|c|c|c|}
\hline \multirow[t]{4}{*}{ Dependent } & \multicolumn{4}{|c|}{ WIN } \\
\hline & \multicolumn{4}{|c|}{ Objectively observable result of penalty shootout: 0 when team loses, 1 when team wins } \\
\hline & \multicolumn{4}{|c|}{ PROBIT REGRESSION } \\
\hline & (1) & (2) & (3) & (4) \\
\hline \multicolumn{5}{|l|}{ Independent } \\
\hline COIN-TOSS-WINNER & $0.5291^{* *}$ & $0.8192 * *$ & $0.8827 * *$ & $1.111^{* *}$ \\
\hline & 0.2606 & 0.3642 & 0.4332 & 0.4577 \\
\hline FIRSTKICK & & 0.2413 & 0.3176 & 0.2241 \\
\hline & & 0.3746 & 0.4295 & 0.4611 \\
\hline FIRSTKICK\#\#DECIDED & & -0.6022 & -0.7880 & -0.7428 \\
\hline & & 0.5294 & 0.6241 & 0.6619 \\
\hline \multicolumn{5}{|l|}{ Further control variables } \\
\hline HOME & & & YES & YES \\
\hline TOURNAMENT & & & YES & YES \\
\hline STAGE & & & YES & YES \\
\hline ATTENDANCE & & & YES & YES \\
\hline POSITION & & & & YES \\
\hline CAPTAIN & & & & YES \\
\hline COACH OLDER & & & & YES \\
\hline GOALKEEPER HEIGHT & & & & YES \\
\hline GOALKEEPER OLDER & & & & YES \\
\hline TEAM OLDER & & & & YES \\
\hline PRIOR SHOOTOUT & & & & YES \\
\hline Evaluation criteria & & & & \\
\hline$N$ & 96 & 96 & 96 & 96 \\
\hline McFadden's $R^{2}$ & 0.032 & 0.042 & 0.148 & 0.224 \\
\hline $\begin{array}{l}\text { Observations correctly } \\
\text { classified }\end{array}$ & $60.42 \%$ & $60.42 \%$ & $67.41 \%$ & $66.67 \%$ \\
\hline Log-Likelihood & -64.356 & -63.637 & -56.641 & -51.582 \\
\hline $\mathrm{BIC}^{\prime}$ & 0.359 & 8.122 & 39.700 & 66.097 \\
\hline
\end{tabular}

Notes: Robust standard errors in bold. We consider only one of the two teams per shootout (i.e., the team listed first officially).

*** Significant at the 1 percent level.

** Significant at the 5 percent level.

* Significant at the 10 percent level.

The definition of variables is described in the main text. 


\section{Figure 1. The effects of winning the coin toss on likelihood of scoring}

\section{Difference in scoring probability between team whose captain won the coin toss and competing team}

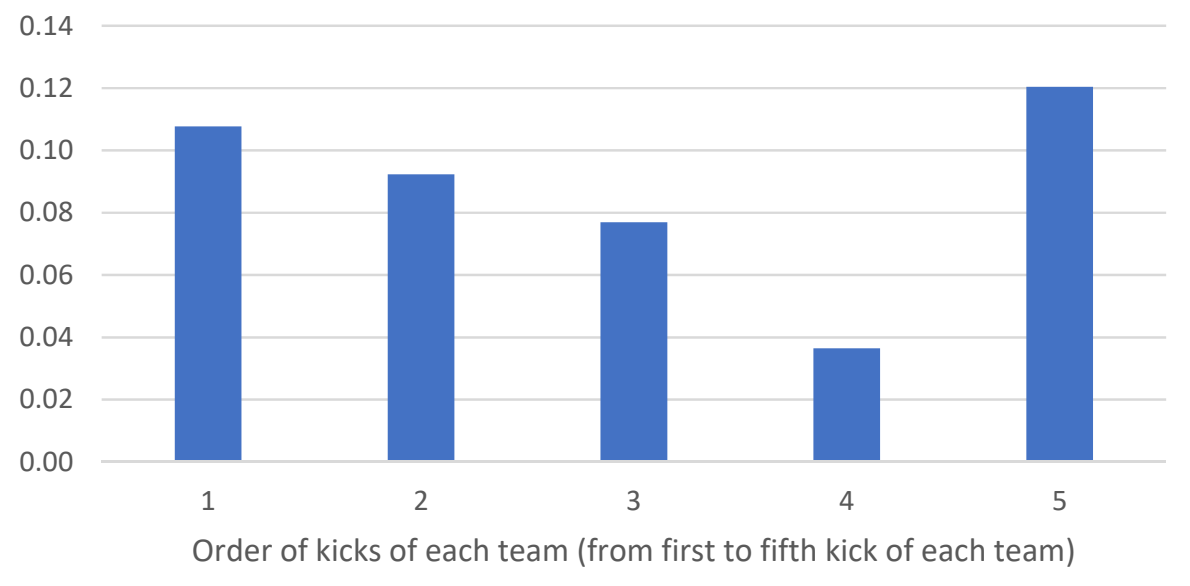

Notes: Overall, the likelihood to score a goal is $72.7 \%$ for the first 10 kicks in the shootouts from tournaments [1] - [9]. The values on the vertical axis are calculated by taking the likelihood to score a goal - conditional on the order of kicks - of the team whose captain won the coin toss and subtract the likelihood to score a goal of the competing team (whose captain lost the coin toss). In this figure, we do not separate the data further in whether the captain who won the coin toss decided to kick first or second. 


\section{Online Appendix}

Table A1. Number of shootouts - with or without knowledge about which captain won the coin toss - conditional on stage of tournament

\begin{tabular}{lcc}
\hline \hline & Known who won the coin toss & Unknown who won the coin toss \\
\cline { 2 - 3 } Final & 13 & 7 \\
Third Place Playoff & 7 & 4 \\
Semi Final & 9 & 12 \\
Quarter Final & 31 & 18 \\
Round of 16 & 19 & 4 \\
Round of 32 & 2 & 66 \\
Previous rounds & 15 & \\
\hline \hline
\end{tabular}


Table A2. Winning frequency of first-kicking team in penalty shootouts from 24 tournaments and seasons $2003 / 2004$ to $2016 / 2017$

\begin{tabular}{|c|c|c|c|c|}
\hline & [A] & [B] & {$[\overline{C C}]$} & [D] \\
\hline Tournament & $\begin{array}{c}\text { Shootouts } \\
\quad N\end{array}$ & $\begin{array}{c}\text { Shootouts with known } \\
\text { order of kicks } \\
N\end{array}$ & $\begin{array}{l}\text { First-kicking team wins } \\
\text { (relative frequency) }\end{array}$ & $\begin{array}{l}\text { P-value (two-sided } \\
\text { binomial test) }\end{array}$ \\
\hline [1] FIFA World Cup & 10 & 10 & 0.8000 & 0.1094 \\
\hline [2] European Championship & 9 & 9 & 0.4444 & 1.0000 \\
\hline [3] Copa America & 11 & 11 & 0.5455 & 1.0000 \\
\hline [4] Africa Cup of Nations & 13 & 13 & 0.3077 & 0.2668 \\
\hline [5] FIFA Confederations Cup & 4 & 4 & 0.2500 & 0.6250 \\
\hline [6] UEFA Champions League & 30 & 30 & 0.6333 & 0.2005 \\
\hline [7] UEFA Europa League & 68 & 68 & 0.4853 & 0.9036 \\
\hline [8] FIFA Club World Cup & 9 & 9 & 0.5556 & 1.0000 \\
\hline [9] FIFA U-20 World Cup & 20 & 20 & 0.4000 & 0.5034 \\
\hline [10] FIFA U-17 World Cup & 10 & 9 & 0.6667 & 0.5078 \\
\hline [11] UEFA U21 Championship & 7 & 7 & 0.5714 & 1.0000 \\
\hline [12] FIFA Women's World Cup & 4 & 4 & 0.7500 & 0.6250 \\
\hline [13] FIFA U-20 Women's World Cup & 6 & 6 & 0.3333 & 0.6875 \\
\hline [14] FIFA U-17 Women's World Cup & 6 & 6 & 0.3333 & 0.6875 \\
\hline [15] UEFA U19 Championship & 6 & 6 & 0.6667 & 0.6875 \\
\hline [16] UEFA U17 Championship & 17 & 14 & 0.3571 & 0.4240 \\
\hline [17] Gold Cup & 7 & 7 & 0.5714 & 1.0000 \\
\hline [18] Asian Cup & 10 & 10 & 0.6000 & 0.7539 \\
\hline [19] DFB Pokal & 104 & 104 & 0.5000 & 1.0000 \\
\hline [20] Premiere Liga Pokal & 5 & 5 & 0.6000 & 1.0000 \\
\hline [21] FA Cup & 43 & 43 & 0.4186 & 0.3604 \\
\hline [22] League Cup & 130 & 129 & 0.4961 & 1.0000 \\
\hline [23] Community Shield & 4 & 4 & 0.5000 & 1.0000 \\
\hline [24] Copa del Rey & 112 & 84 & 0.5238 & 0.7436 \\
\hline All & 645 & 612 & 0.5016 & 0.9678 \\
\hline
\end{tabular}

